\title{
ESTUDO DA ADSORÇÃO DE COMPOSTOS SULFURADOS EMPREGANDO ZEÓLITAS CONTENDO ZINCO
}

\author{
Álvaro L. Malvesti, Marcelo L. Mignoni, Robison P. Scherer, Fábio G. Penha e Sibele B. C. Pergher* \\ Departamento de Química, Universidade Regional Integrada do Alto Uruguai e das Missões, Av. Sete de Setembro, 1621, \\ 99700-000 Erechim - RS, Brasil
}

Recebido em 22/7/08; aceito em 11/2/09; publicado na web em 3/7/09

\begin{abstract}
STUDY OF ADSORPTION OF SULPHUR COMPOUNDS EMPLOYING ZEOLITES CONTAINING ZINC. This paper deals with an adosrption of sulphur compounds employing zeolites containing zinc. The zeolites employed were commercial NaY and Beta. The zinc was incorporated in three levels: $0.5 ; 1.0$ and $5 \%$. The sulphur compounds studied were benzothiophene and dibenzothiofene. The results showed that both zeolites can be employed for adsorption of benzothiophene and dibenzothiophene. The $\mathrm{Zn}$ incorporation $(0.5 \%)$ promotes an increase in zeolites adsorption ability. The DBT adsorbs more than BT, probably because it strongly interacts with zeolite structure. The BT adsorbs more in NaY than in beta probably because the NaY zeolite has a high intern volume. This is not observed for DBT.
\end{abstract}

Keywords: sulphur compounds; zeólitas; adsorption.

\section{INTRODUÇÃ̃o}

A presença de compostos sulfurados em frações de petróleo é altamente indesejável devido sua ação corrosiva e poluição atmosférica, promovida por gases prejudiciais ao meio ambiente, gerados durante a combustão.

Assim, a necessidade de emissões cada vez mais limpas resulta na constante busca de redução de enxofre nos combustíveis. Durante o processo de combustão, o enxofre reage com o oxigênio para formar $\mathrm{SO}_{2}$ e $\mathrm{SO}_{3} \mathrm{O} \mathrm{SO}_{3}$, por sua vez, a partir de sua reação com $\mathrm{H}_{2} \mathrm{O}$ forma $\mathrm{O}_{2} \mathrm{SO}_{4}$, que é extremamente nocivo às partes metálicas de equipamentos podendo levar a altíssimas taxas de corrosão e formação de chuva ácida. ${ }^{1}$

Visando uma redução de compostos sulfurados, tem-se buscado o desenvolvimento de tecnologias para a remoção de enxofre de combustíveis. Os processos de hidrotratamento são bastante utilizados, além de catalisadores sofisticados de cobalto e molibdênio, resultando no alto custo da eliminação do teor de enxofre. Entretanto, um processo alternativo ao hidrotratamento é a adsorção, por ter condição de operação menos severa e mais econômica.

A aplicação das zeólitas na indústria do controle ambiental está se tornando cada vez mais importante, sobretudo devido ao maior conhecimento de suas propriedades e características. Sendo um material poroso, as zeólitas possuem uma grande área superficial oferecendo ao processo de adsorção uma elevada área de transferência de massa. ${ }^{2}$

Ainda, sabe-se que alguns metais como, por exemplo, o zinco apresentam afinidade com o S, formando compostos complexos que propiciam a adsorção de compostos sulfurados. Na literatura encontram-se trabalhos empregando zeólitas com $\mathrm{Cu},{ }^{3-7} \mathrm{Ni},{ }^{3-6} \mathrm{Ag},{ }^{3,5}$ $\mathrm{Zn},{ }^{3-5} \mathrm{Fe}^{4,5}$ para adsorção de compostos sulfurados como benzotiofeno, dibenzotiofeno, tiofeno, entre outros. Também há trabalhos empregando zirconia,${ }^{8}$ sílicas mesoporosas, ${ }^{9}$ zeólitas sintetizadas a partir de cinzas de carvão, ${ }^{10}$ zeólitas sem a incorporação de metais ${ }^{11,12}$ e zeólitas com substituição isomórfica de Ga na estrutura. ${ }^{13}$ Estes trabalhos empregam vários solventes, como diesel e gasolina.

Neste contexto, o objetivo deste trabalho, foi estudar a remoção de compostos de enxofre, utilizando como adsorventes a zeólita NaY e Beta, impregnadas com zinco.

*e-mail: pergher@uri.com.br
A estrutura da zeólita Beta está formada pelo intercrescimento de dois polimorfos (A e B) muito relacionados entre si, com a possibilidade que exista um terceiro (C). Os polimorfos são constituídos por um sistema tridirecional de canais delimitados por anéis de 12 membros e estão interconectados. (A Figura 1S do material suplementar representa estes polimorfos A, B e C)..$^{14}$

A outra zeólita empregada foi a zeólita $\mathrm{NaY}$, que apresenta poros com aberturas de 12 membros (12MR) (esta estrutura está esquematizada na Figura 2S, material suplementar). Esta zeólita apresenta dois sistemas de canais interconectados entre si: um sistema formado pela união de supercaixas $\alpha$ (diâmetro interno de $12,4 \AA$ ), à qual se ingressa por aberturas formadas por $12 \mathrm{MR}$ de diâmetro igual a 7,8 $\AA$; um sistema de canais formado pela conexão alternada de caixas sodalitas (diâmetro interno igual a 6,6 ̊) e supercaixa $\alpha$, à qual se penetra por aberturas de $6 \mathrm{MR}$ de diâmetro igual a 2,2 $\mathrm{A}$.

\section{PARTE EXPERIMENTAL}

\section{Materiais adsorventes}

Os materiais empregados neste estudo foram, as zeólitas Beta e $\mathrm{NaY}$, fornecidas pelo Instituto de Tecnologia Química da Universidade Politécnica de Valencia (Espanha).

\section{Impregnação de zinco}

As zeólitas Beta e NaY foram impregnadas com zinco em diferentes concentrações em peso $0,5,1$ e $5 \%$, utilizando solução de $\mathrm{ZnCl}_{2}$, em refluxo com temperatura de $80^{\circ} \mathrm{C}$ por $4 \mathrm{~h}$. Posteriormente, os materiais foram secos em rota-vapor e em estufa por $12 \mathrm{~h}$ a $110^{\circ} \mathrm{C}$.

\section{Teste de adsorção de compostos sulfurados}

Os testes de adsorção foram realizados empregando-se $100 \mathrm{mg}$ de adsorvente em $20 \mathrm{~mL}$ de uma solução contendo $1000 \mathrm{mg} / \mathrm{L}$ de benzotiofeno (Aldrich, 98\%) ou dibenzotiofeno (Aldrich, 98\%) em ciclo-hexano (combustível sintético), em temperatura ambiente, com agitação constante e em diferentes tempos de contato (1 a 24 h). A análise quantitativa do benzotiofeno e dibenzotiofeno foi realizada num GC/MS (cromatógrafo gasoso/acoplado ao espectro de massas, 
QP5050A, Shimadzu, com autoinjetor AOCi). A Tabela 1 apresenta as condições utilizadas para a análise. Empregaram-se os compostos de enxofres: benzotiofeno e dibenzotiofeno (Figura 3S, material suplementar), pois estes tipos de compostos, normalmente estão presentes em combustíveis.

Tabela 1. Condições de análises para o GC/MS

\begin{tabular}{lc}
\hline Parâmetros & Condições \\
\hline Temperatura inicial & $50{ }^{\circ} \mathrm{C}$ \\
Tempo & $2 \mathrm{~min}$ \\
Rampa de aquecimento & $8{ }^{\circ} \mathrm{C} / \mathrm{min}$ \\
Temperatura final & $300{ }^{\circ} \mathrm{C}$ \\
Tempo final & $10 \mathrm{~min}$ \\
Coluna & DB-5 $(30 \mathrm{~m}$ x $0,25 \mathrm{~mm} \times 0,25 \mu)$ \\
Gás de arraste & hélio \\
(fluxo constante) & split $(1: 3)$ \\
Modo de injeção & $2 \mathrm{~mL} / \mathrm{min}$ \\
Velocidade linear & variável \\
do gás de arraste & $44,5 \mathrm{~min}$ \\
Pressão da coluna & \\
Tempo de corrida &
\end{tabular}

\section{Caracterização dos materiais}

Os materiais preparados, bem como as zeólitas, foram caracterizados por diferentes técnicas: difração de raios-X, espectroscopia de fluorescência de raios-X e análise textual por adsorção de $\mathrm{N}_{2}$

As análises de difração de raios $\mathrm{X}$ foram realizadas num diffraktometer modelo D5000 (Siemens) utilizando filtro de Ni e radiação $\mathrm{Cu}-\mathrm{k} \alpha(\lambda=1,54 \AA)$.

A caracterização textural dos materiais foi realizada utilizando um autosorb-1 da Quantachrome (Nova-2200e). Antes da análise, cerca de $100 \mathrm{mg}$ de amostra foram tratadas a vácuo, a uma temperatura de $300^{\circ} \mathrm{C}$ por $3 \mathrm{~h}$. As medidas foram realizadas na temperatura do $\mathrm{N}_{2}$ líquido.

A análise química foi realizada por fluorescência de raios X (FRX) utilizando-se um espectro de raios X Philips modelo PW 1480.

\section{RESULTADOS E DISCUSSÃO}

\section{Caracterização das zeólitas}

O difratograma de raios- $\mathrm{X}$ obtido para a zeólita Beta está representado na Figura 1. Ao compará-lo com dados descritos na literatura, ${ }^{14}$ pode-se observar que se trata do material zeolítico Beta pura e cristalina, ou seja, sem contaminação de outras fases zeolíticas e ordenado estruturalmente.

A Figura 2 ilustra o difratograma de raios-X obtido para a zeólita NaY. Observa-se ao comparar o difratograma com os dados de literatura, ${ }^{15}$ que se trata da zeólita NaY (tipo Faujasita).

As isotermas de adsorção de nitrogênio mostram que os materiais são microporosos (isoterma do tipo I). A partir destes dados de adsorção foram calculadas as áreas superficiais específicas empregando a equação BET $^{16}$ e estes resultados, bem como o da análise química realizado por espectroscopia de fluorescência de raios-X, estão descritos na Tabela 2.

Pode-se observar que a zeólita Beta apresenta uma maior área superficial em relação à zeólita $\mathrm{NaY}$, assim como uma maior relação molar $\mathrm{SiO}_{2} / \mathrm{Al}_{2} \mathrm{O}_{3}$.

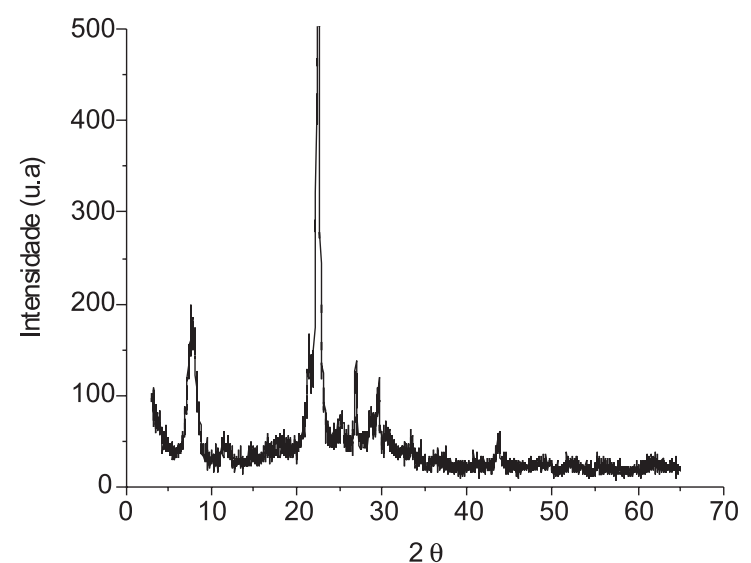

Figura 1. Difratograma de raios-X da zeólita Beta

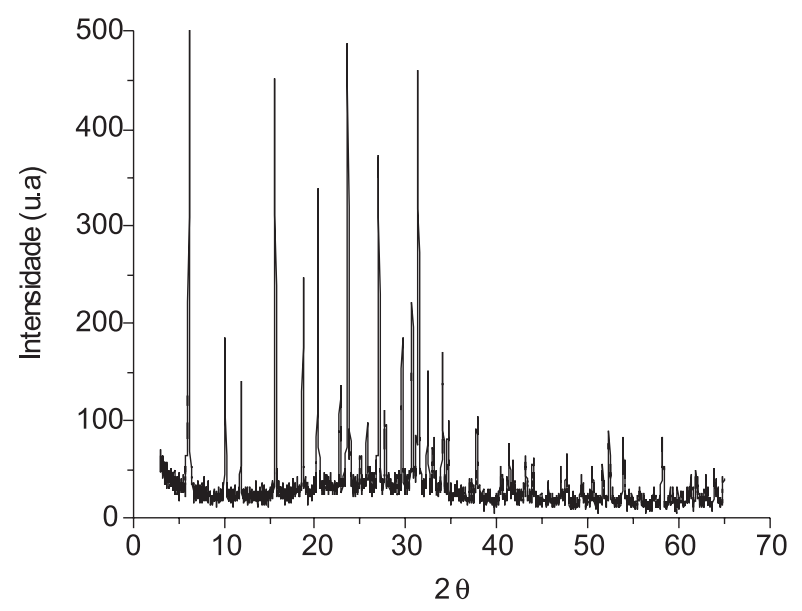

Figura 2. Difratograma de raios- $X$ da zeólita $\mathrm{NaY}$

Tabela 2. Características físicas e químicas das zeólitas

\begin{tabular}{lcc}
\hline Zeólitas & $\begin{array}{c}\text { Área superficial } \\
\mathrm{BET}\left(\mathrm{m}^{2} / \mathrm{g}\right)\end{array}$ & $\begin{array}{c}\text { Relação molar } \\
\mathrm{SiO}_{2} / \mathrm{Al}_{2} \mathrm{O}_{3}\end{array}$ \\
\hline Beta & 730 & 25 \\
$\mathrm{NaY}$ & 568 & 5,2 \\
\hline
\end{tabular}

Testes de adsorção do benzotiofeno

A Figura 3 mostra a cinética de adsorção para as diferentes zeólitas Beta impregnadas com Zn. Observa-se que com o aumento do tempo ocorre uma diminuição no teor de benzotiofeno adsorvido, provavelmente pela ocorrência do processo de saturação causado pelo acréscimo de tempo de contato das soluções de benzotiofeno com os adsorventes.

Este resultado não era o esperado, uma vez que, de acordo com a literatura, ${ }^{17} \mathrm{o} \mathrm{Zn}$ incorporado em zeólitas forma complexos com compostos de $\mathrm{S}$, alcançando o equilíbrio na adsorção com o tempo. Entretanto, ao compararmos os resultados obtidos com trabalhos anteriores, observamos que os mesmos trabalham com tempos menores (até $1 \mathrm{~h}$ ) e que empregam como composto de $\mathrm{S}$ o propanotiol, que é uma molécula menor que o benzotiofeno. Entretanto, esta última é a que normalmente se encontra em combustíveis. Apesar de ocorrer uma diminuição da adsorção com o tempo, comparando os resultados obtidos nas primeiras $4 \mathrm{~h}$, observa-se a seguinte ordem decrescente de adsorção: $\mathrm{ZnB}-0,5 \%>\mathrm{ZnB}-1 \%>$ Beta pura $>\mathrm{ZnB}$ $5 \%$. Indicando que ao impregnar $\mathrm{Zn}$ na zeólita até a quantidade de 
$1 \%$ de $\mathrm{Zn}$, aumenta a capacidade de adsorção. Entretanto, em teores maiores que $1 \%$ de $\mathrm{Zn}$ a capacidade de adsorção diminui. Isto ocorre provavelmente por obstrução dos poros, diminuindo desta forma a acessibilidade da molécula, ou ocorre sinterização, diminuindo a área de Zn disponível.

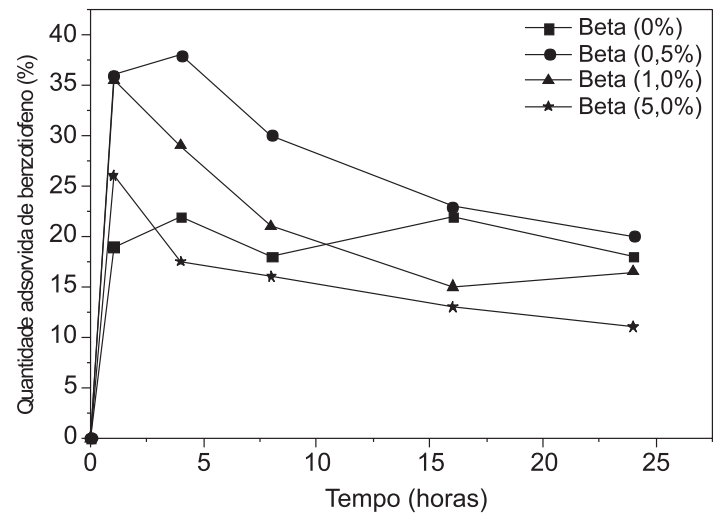

Figura 3. Cinética de adsorção de benzotiofeno utilizando zeólita Beta pura, $\mathrm{ZnB}-0,5 \%, \mathrm{ZnB} 1 \%$ e $\mathrm{ZnB}-5 \%$

Na Figura 4 apresenta-se o gráfico de adsorção do benzotiofeno empregando os diferentes materiais preparados com a zeólita Y. Observa-se que em tempos iniciais ocorre uma grande adsorção de benzotiofeno e com o tempo esta quantidade diminui, atingindo o equilíbrio. Esta queda é mais evidente na zeólita Y sem $\mathrm{Zn}$ incorporado. Observa-se para tempos maiores que $4 \mathrm{~h}$ que o material com $0,5 \%$ de $\mathrm{Zn}$ incorporado é melhor adsorvente e que quando se aumenta o teor de $\mathrm{Zn}$ incorporado ocorre diminuição da adsorção do benzotiofeno ou, também, como descrito pode ocorrer sinterização. Resultados apontam que com o aumento do teor de $\mathrm{Zn}$ deve estar ocorrendo um bloqueio dos poros, o que é mais bem evidenciado com o benzotiofeno por ser uma molécula maior que o propanotiol. Como foi evidenciado também para a zeólita Beta.

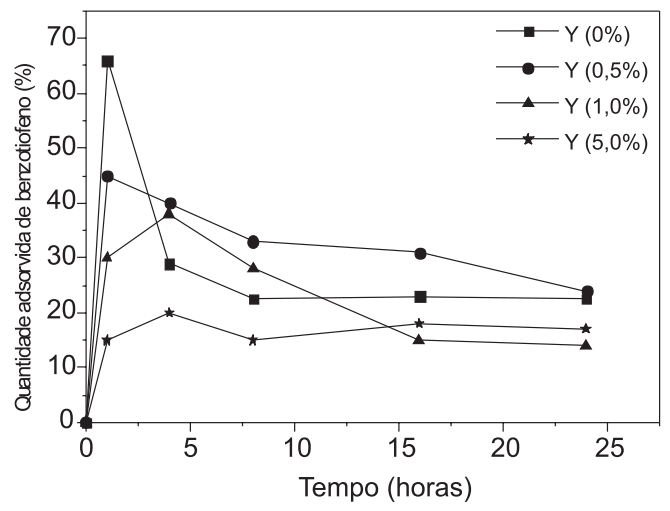

Figura 4. Cinética de adsorção do benzotiofeno em zeólita NaY pura e com 0,5; 1,0 e 5,0\% de Zn incorporado

A Figura 5 compara as duas zeólitas $\mathrm{NaY}$ e Beta impregnadas com $0,5 \%$ de $\mathrm{Zn}$ na adsorção do benzotiofeno. Observa-se que a zeólita $\mathrm{NaY}$ adsorve maiores quantidades de benzotiofeno que a Beta. Ambas as zeólitas são acessíveis por poros de 12MR, embora a NaY tenha área superficial menor que a da Beta $\left(568 \mathrm{~m}^{2} / \mathrm{g}<730 \mathrm{~m}^{2} / \mathrm{g}\right)$, ela possui cavidades que proporcionam maiores volumes interiores favorecendo uma maior adsorção do benzotiofeno. Isso indica que a estrutura zeolítica influi no processo de adsorção.

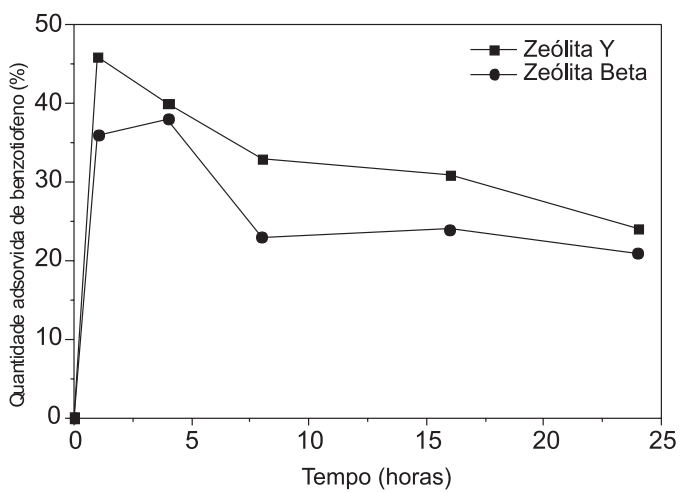

Figura 5. Comparação entre as duas zeólitas NaY e Beta impregnadas com 0,5\% de Zn na adsorção do benzotiofeno

\section{Testes de adsorção do dibenzotiofeno}

Testes empregando dibenzotiofeno foram realizados nas zeólitas Beta e NaY impregnadas somente com 0,5\% de $\mathrm{Zn}$, uma vez que este foi o teor de zinco que apresentou melhor resultado para o benzotiofeno.

A Figura 6 mostra a cinética de adsorção do dibenzotiofeno para as zeólitas Beta e NaY pura. Ao comparar estes resultados com os anteriores para o benzotiofeno, observa-se uma maior adsorção do dibenzotiofeno. Apesar da molécula do dibenzotiofeno ser maior do que a do benzotiofeno e provavelmente ter mais dificuldades de se difundir nas cavidades zeolíticas, sua maior adsorção devese a uma maior interação com a superfície zeolítica. A molécula dibenzotiofeno possui dois anéis tiofeno, enquanto que a molécula do benzotiofeno possui somente um anel tiofeno. Os anéis tiofeno interagem com os cátions da zeólita formando complexos- $\pi$ através da doação eletrônica $\sigma-\pi .^{6}$

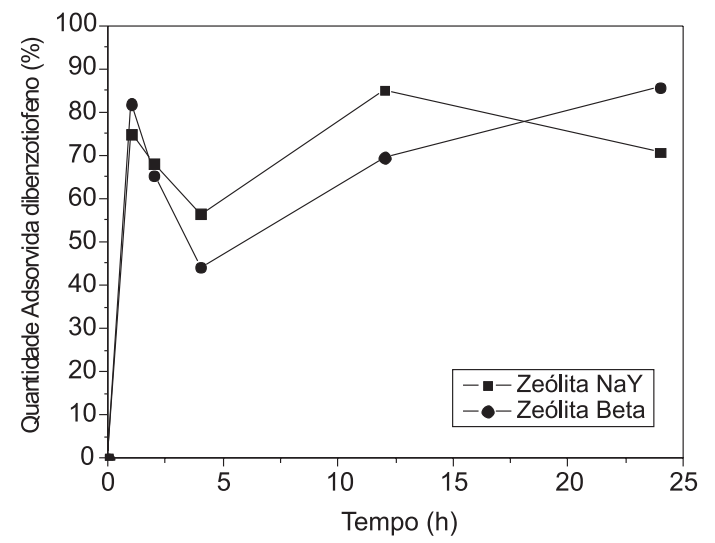

Figura 6. Cinética de adsorção de dibenzotiofeno utilizando as zeólitas beta e NaY

Ambas zeólitas adsorvem quantidades similares de dibenzotiofeno e não se observa influência da estrutura zeolítica, como observado para o benzotiofeno.

A Figura 7 apresenta a cinética de adsorção para as zeólitas NaY e Beta impregnadas com 0,5\% de Zn. Ambas zeólitas impregnadas com $\mathrm{Zn}$ adsorvem quantidades similares. Ao se comparar estes resultados com os anteriores sem a incorporação de zinco, observa-se um aumento na quantidade adsorvida com a impregnação de zinco.

Os difratogramas de raios- $\mathrm{X}$ obtidos para as zeólitas Beta e NaY impregnadas com zinco $0,5 \%$ em peso (Figuras $4 \mathrm{~S}$ e $5 \mathrm{~S}$, material suplementar) são idênticos aos difratogramas das zeólitas pura, indicando que a impregnação do zinco não destruiu a estrutura da zeólita. 


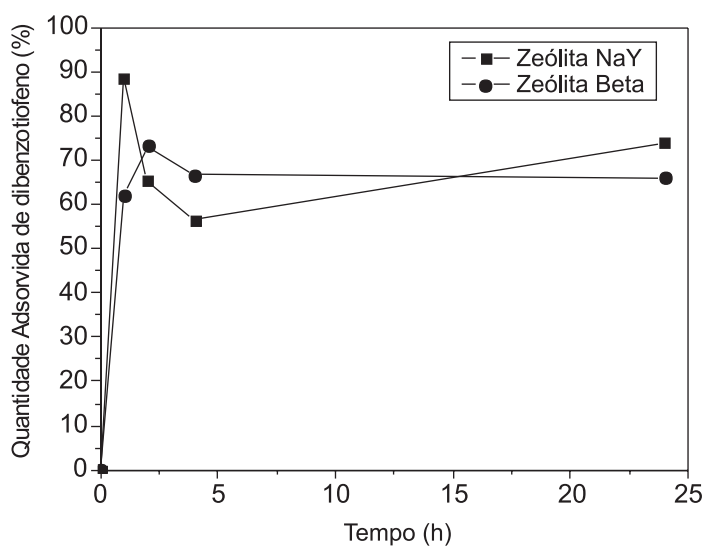

Figura 7. Cinética de adsorção de dibenzotiofeno utilizando as zeólitas beta e NaY com $0,5 \%$ de $\mathrm{Zn}$

\section{CONCLUSÕES}

As zeólitas Beta e NaY são bons adsorventes para compostos sulfurados como benzotiofeno e dibenzotiofeno. A incorporação de $\mathrm{Zn}$ em teores de $0,5 \%$ não destruiu a estrutura zeolítica e proporcionou melhora na capacidade de adsorção de benzotiofeno e dibenzotiofeno. O composto dibenzotiofeno adsorve em quantidades maiores que o benzotiofeno, provavelmente por sua interação mais forte com a estrutura zeolítica. O composto benzotiofeno é adsorvido em quantidades maiores na $\mathrm{NaY}$ do que na Beta, provavelmente pelo maior volume interno desta zeólita, entretanto este fato não é observado para o dibenzotiofeno. Estes resultados são promissores no que se refere à remoção de compostos sulfurados de combustíveis, que normalmente apresentam compostos como benzotiofeno e dibenzotiofeno.

\section{MATERIAL SUPLEMENTAR}

No material suplementar, disponível gratuitamente em http:// quimicanova.sbq.org.br, na forma de aquivo PDF, encontram-se as Figuras $1 \mathrm{~S}$ a $5 \mathrm{~S}$.

\section{REFERÊNCIAS}

1. Ushima, A. H.; Filho, R. V.; Resumo do 30 Workshop de combustão e Propulsão, Lorena, Brasil, 1989.

2. Lima, E. C.; Barbosa, C. M. B. M.; Aguilar, E. F. S.; Barros, A. L. M.; Resumos do $2^{\circ}$ Congresso Brasileiro de P\&D em Petróleo \& Gás, Rio de Janeiro, Brasil, 2003.

3. Zhang, Z. Y.; Shi, T. B.; Jia, C. Z.; Ji, W. J.; Chen, Y.; He, M. Y.; Appl. Catal., $B$ 2008, 82,1 .

4. Bhandari, V. M.; Ko, C. H.; Park, J. G.; Han, S.; Cho, S.; Kim, J.; Chem. Eng. Sci. 2006, 61, 2599.

5. Yu, M.; Li, Z.; Xia, Q.; Xi, H.; Wang, S.; Chem. Eng. J. 2007, 132, 233.

6. Jiang, M.; Ng, F. T. T.; Catal. Today 2006, 116, 530.

7. King, D. L.; Li, L.; Catal. Today 2006, 116, 526.

8. Baeza, P.; Aguila, G.; Gracia, F.; Araya, P.; Catal. Commun. 2008, 9, 751.

9. Park, J. G.; Ko, C. H.; Yi, K. B.; Park, J.; Han, S.; Cho, S.; Kim, J.; Appl. Catal., B 2008, 81, 244.

10. Ngamcharussruvuchai, C.; Chatratananon, C.; Nuntang, S.; Prasassarakich, P.; Fuel 2008, 87, 2347.

11. Jiang, M.; Ng, F. T. T.; Rahaman, A.; Patel, V.; Thermochim. Acta 2005, 434, 27.

12. Gutiérrez-Alejandre, A.; Larrubia, M. A.; Ramirez, J.; Busca, G.; Vib. Spectrosc. 2006, 41, 42.

13. Tang, K.; Song, L.; Duan, L.; Li, X.; Gui, J.; Sun, Z.; Fuel Process. Technol. 2008, 89, 1 .

14. Valencia, S. V.; Tese de Doutorado, Universidade Politécnica de Valencia, Espanha, 1997.

15. Ballmoos, R. V.; Collection of Simulated XRD Powder Pattems For Zeolites, Butterwortth: U.K., 1987.

16. Giannetto, G. P.; Zeólitas: Caracteristicas, Propriedades y Aplicaciones Industrials, Ediciones Innovación Tecnológica: Venezuela, 1990.

17. Clericuzi, G. Z.; Barbosa, C. M. B. M.; Souza-Aguiar, E. F.; Medina, N.; Resumos do $2^{\circ}$ Congresso Brasileiro de P\&D em Petróleo \& Gás, Rio de Janeiro, Brasil, 2003. 
Álvaro L. Malvesti, Marcelo L. Mignoni, Robison P. Scherer, Fábio G. Penha e Sibele B. C. Pergher*

Departamento de Química, Universidade Regional Integrada do Alto Uruguai e das Missões, Av. Sete de Setembro, 1621, 99700-000 Erechim - RS, Brasil

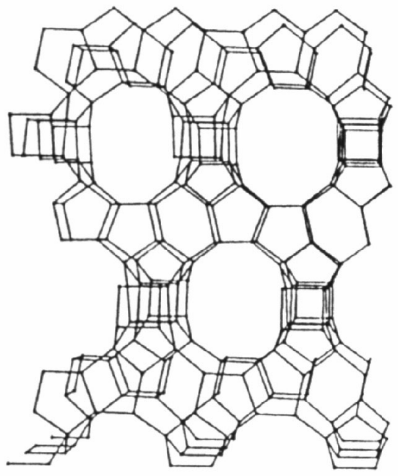

A

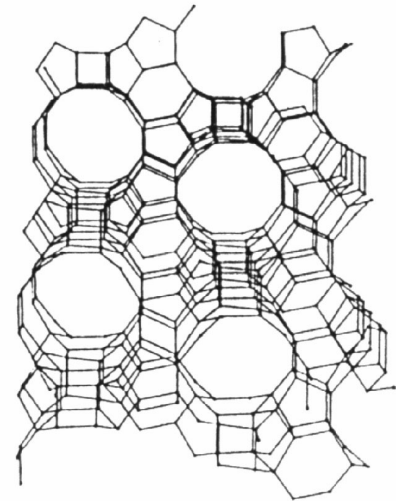

B

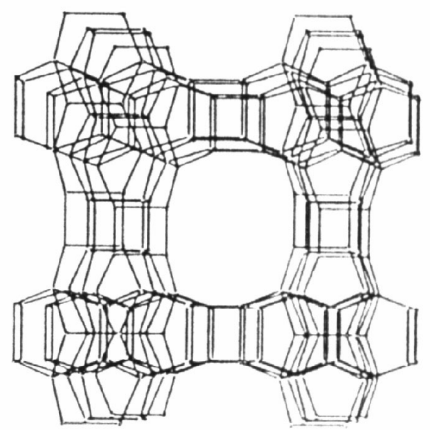

C

Figura 1S. Polimorfos A, B e C da zeólita Beta

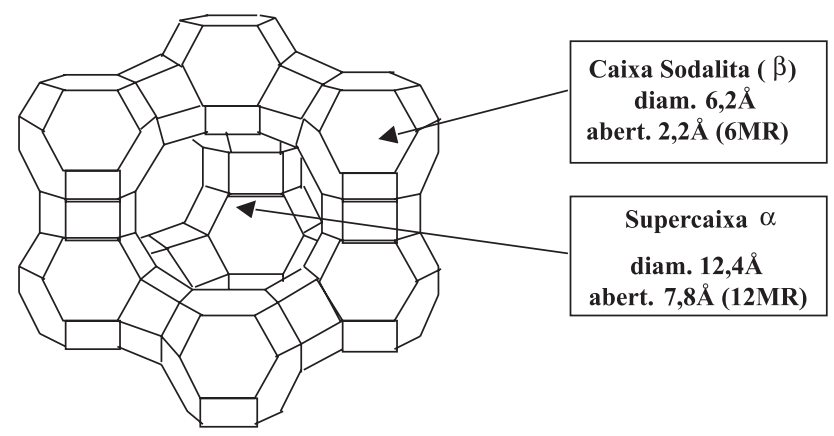

Figura 2S. Estrutura demonstrativa da zeólita $\mathrm{NaY}$ 
(a)

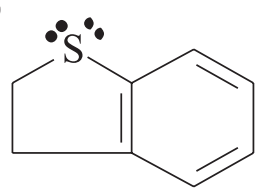

(b)<smiles>C1=CC2c3ccccc3S[C@@H]2C=C1</smiles>

Figura 3S. Compostos sulfurados: (a) benzotiofeno e (b) dibenzotiofeno

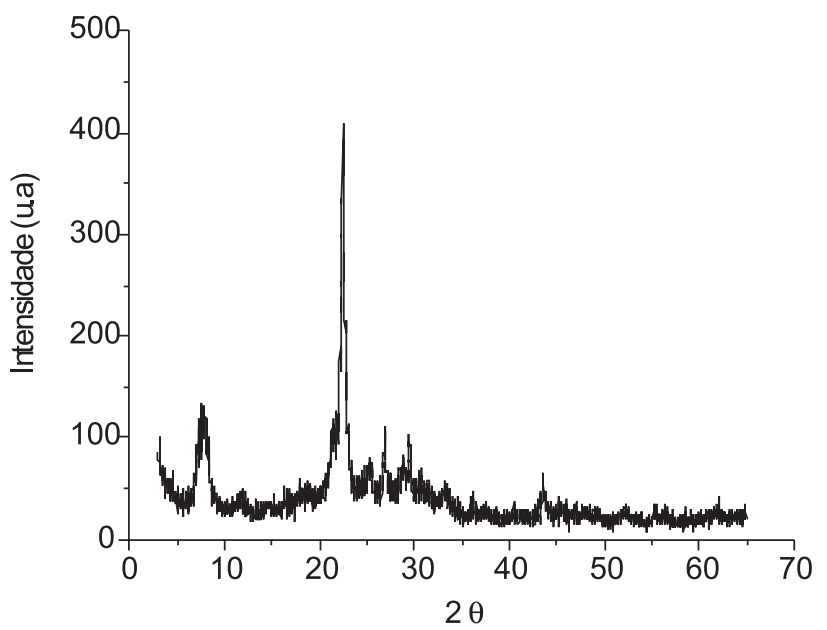

Figura 4S. Difratograma de raios- $X$ da zeólita Beta com zinco

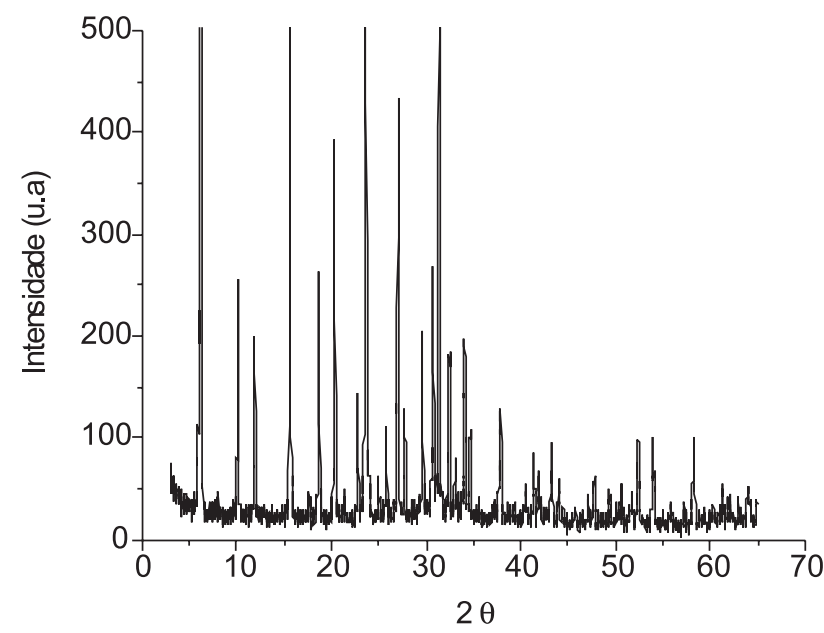

Figura 5S. Difratograma de raios-X da zeólita NaY com zinco 